\title{
Inventaire et caractérisation des plantes médicinales utilisées en thérapeutique dans le département de la Sanaga Maritime: Ndom, Ngambe et Pouma
}

\author{
Emmanuel Nnanga Nga (1,2), Catherine KIDIK POUKA¹, Philomène Céleste NGO BOUMSONG1', Siegfried \\ Didier DIBONG*(1,3), Emmanuel MPONDO MPONDO(1,4) \\ ${ }_{1}$ Département des Science, Pharmaceutiques, Faculté de Médecine et des Sciences Pharmaceutiques, Université de \\ Douala, B.P. 2701 Douala, Cameroun \\ ${ }^{2}$ Département de Galénique, Faculté de Médecine et des Sciences Biomédicales, Université de Yaoundé I, B.P. 1364 \\ Yaoundé, Cameroun \\ ${ }^{3}$ Département de Biologie des Organismes Végétaux, Faculté des Sciences, B.P. 24157 Douala, Cameroun \\ ${ }^{4}$ Département de Pharmacotoxicologie et Pharmacocinétique, Faculté de Médecine et des Sciences Biomédicales, \\ Université de Yaoundé I, B.P. 1364 Yaoundé, Cameroun \\ * Auteur de la correspondance : didierdibong@yahoo.fr
}

Original submitted in on $1^{\text {st }}$ September 2016. Published online at www.m.elewa.org on $31^{\text {st }}$ October 2016 http://dx.doi.org/10.4314/jab.v106i1.13

\section{RESUME}

Objectif : Dans le but de contribuer à la connaissance des plantes médicinales utilisées pour les traitements des symptômes qui affectent les populations rurales du département de la Sanaga maritime, une enquête ethnobotanique et ethnopharmacologique a été réalisée.

Méthodologie et Résultats : L'étude réalisée auprès de 94 ménages a permis de recenser 94 espèces végétales appartenant à 92 genres et 54 familles. Les familles les plus représentées ont été les Fabaceae, Euphorbiaceae, et les Asteraceae, avec respectivement 9 et 6 espèces. Dix genres ont été majoritaires à savoir : Aframomum, Alchornea, Allium, Carica, Citrus, Entandrophragma, Ocimum, Solanum, Urera et Vernonia dans les proportions de $14 \%$ pour Allium et Ocimum et $9 \%$ pour les autres genres. Les paramètres écologiques des espèces végétales ont permis d'identifier 12 types de biotopes avec $28,85 \%$ d'espèces cultivées et $21,15 \%$ d'espèces de forêts primaires. Seize types phytogéographiques recensé soit $24,04 \%$ de pantropicales, $20,19 \%$ d'Afro tropicales et 19,23\% de Guinéo congolaises. Les espèces exploitées ont été en majorité les herbacées soit $37,50 \%$ suivis des arbres et arbustes avec respectivement $29,81 \%$ et $27,88 \%$. Les sarcochores et les sclérochores ont été les types de diaspores majoritaires avec respectivement $43,27 \%$ et $13,46 \%$. La décoction et la macération ont été les modes de préparation les plus utilisés par les tradipraticiens avec $27,46 \%$ et $23,94 \%$. La voie orale était la plus utilisée pour l'administration des recettes de plantes médicinales. Quatre-vingt recettes ont été retenues pour le traitement des symptômes affectant les populations de ce département. Le facteur de consensus calculé a permis de confirmer les troubles dus aux protozoaires et les mycoses comme étant celles qui sévissent le plus dans la région avec des indices respectifs de 0,71 et 0,50 . Les alcaloïdes, flavonoïdes, coumarines, phénols, tanins, saponosides et terpènes ont été révélés comme métabolites secondaires. Les feuilles représentaient l'organe le plus utilisé pour les recettes. 


\section{Nga et al. J. Appl. Biosci. $2016 \quad$ Inventaire et caractérisation des plantes médicinales utilisées en}

thérapeutique dans le département de la Sanaga Maritime : Ndom, Ngambe et Pouma

Conclusion et Application des résultats: Les plantes médicinales recensées, aux métabolites secondaires variés constituent un atout indéniable dans la fabrication des médicaments traditionnels améliorés.

Mots clés : plantes médicinales, ethnobotanique, ethnopharmacologie, métabolites secondaires.

\section{ABSTRACT}

Objective: In order to contribute to the knowledge of medicinal plants used for diseases treatments and symptoms affecting rural populations of the Sanaga Maritime division, ethnobotanical and ethno pharmacological investigations have been carried.

Methodology and results: Only people aged between 29-70 years with a confirmed knowledge of medicinal plants and their usage in curing and willing to take part of the study have been interviewed. The study realized in 94 homes allowed making an inventory of 94 vegetable species belonging to 92 genera and 54 families. The most representative were Fabaceae, Euphorbiaceae and Asteraceae respectively 9 and 6 for the two others. Among those species, 10 genera have been the most representative namely: Aframomum, Alchornea, Allium, Carica, Citrus, Entandrophragma, Ocimum, Solanum, Urera and Vernonia in the following proportions $14 \%$ for Allium and Ocimum and $9 \%$ for others. Ecological parameters of plants species allowed the identification of 12 types of biotopes with $28.85 \%$ species harvested and $21.15 \%$ species of primary forests. Sixteen phytogeographical types have been listed to with $24.04 \%$ of "pantropicales", $20.19 \%$ "Afro tropicales" and $19.23 \%$ "guineo congolaises". Exploited species have been in majority herbaceous $37.50 \%$, followed respectively by trees and shrubs with $29.81 \%$ and $27.88 \%$. Sarcochores and Slerochores were the high majority of diaspores with the following proportions $43.27 \%$ and $13.46 \%$. Decoction and maceration have been the preparation method most used by traditional practitioners with $27.46 \%$ and $23.94 \%$. The oral route was the most used for the administration of medicinal plants recipes. 80 recipes have been retained for the treatment of diseases and symptoms affecting people of this division. The consensus factor permitted to confirm that troubles caused by protozoa and mycoses were raging in that division with 0.71 and 0.50 respectively. Have been identified as secondary metabolites: alkaloids, flavonoids, coumarins, phenols, tannins, saponins and terpenoids. Leaves represented the most used part for the preparation of recipes.

Conclusion and applications of results: The usage of medicinal plants by African people and others in the world for the multiple diseases pushed researchers to explore this way to find new molecules.

Keys words: medicinal plants, ethnobotanic, ethnopharmacology, secondary metabolites.

\section{INTRODUCTION}

Les plantes, éléments vitaux de la diversité biologique, servent essentiellement au bien être humain. En dehors des plantes cultivées, plusieurs plantes sauvages peu connues revêtent une grande importance culturelle et un fort potentiel économique pour l'alimentation, les soins, l'énergie, l'habillement et la construction des logements (Koné et Kamanzi, 2006). Les relations entre les plantes et les hommes existent depuis de longues dates (Chikamai et al., 2009). Environ $80 \%$ des cinq milliards d'habitants que compte la planète à recours à la médecine traditionnelle (Mbita Messi, 1998). Les plantes médicinales constituent des ressources précieuses pour la majorité des populations rurales en Afrique (Jiofack et al., 2010). De plus, les produits forestiers non ligneux (PFNLs) ont éveillé un intérêt considérable en Afrique au cours de ces dernières années pour leur contribution à l'économie des ménages et la conservation de la biodiversité végétale (Betti, 2002). Par ailleurs, ces plantes constituent des ressources inestimables pour l'industrie pharmaceutique (Awono et al., 2009). Au Cameroun, des études ont révélé que les médicaments à base de plantes seraient considérés comme peu toxiques par rapport aux médicaments de synthèse (Dibong et al., 2011). Les plantes médicinales constituent des ressources précieuses pour la grande majorité des populations rurales en Afrique (Fondoum et Tiki Manga, 2000). Au Cameroun, la majeure partie de la population n'a pas 
accès aux soins de santé moderne, raison pour laquelle l'utilisation des plantes médicinales en thérapeutique est très répandue. La pharmacopée camerounaise est riche en raison de la diversité des groupes humains, des langues, des coutumes et surtout des caractéristiques écologiques des régions. Les différents systèmes de soins mis en place, ont fait preuve d'une efficacité durable et inoffensive pour la santé de l'Homme, mais aussi des animaux domestiques (Guedje et Fankap, 2001). Dans la région du Littoral, l'exploitation des produits forestiers non ligneux intéresse de nombreuses personnes démunies et sans-emploi (Priso et al., 2011). Dans le département de la Sanaga Maritime, notamment les arrondissements

\section{MATERIEL ET METHODES}

Situation géographique : L'étude a été menée dans 3 arrondissements du département de la Sanaga maritime et a requis un certain matériel. La méthodologie utilisée a conduit à des résultats qui seront analysés. La Sanaga maritime est un département du Cameroun situé dans la région du littoral, avec pour chef-lieu Edéa (latitude $4^{\circ}$ $30^{\prime}-4^{\circ} 35^{\prime} \mathrm{N}$; longitude $10^{\circ} 50^{\prime}-10^{\circ} 55^{\prime} \mathrm{E}$ ) qui s'étend sur une superficie de $18000 \mathrm{ha}\left(180 \mathrm{~km}^{2}\right)$. Le climat d'Edéa appartient au domaine équatorial de type guinéen qui se caractérise par quatre saisons : une grande saison de pluie (de mi-août à mi-novembre), une grande saison sèche (de mi-novembre à février), une petite saison de pluie (de mars à mai) et une petite saison sèche (entre juillet et mi-août), des précipitations annuelles moyennes (entre 1500 et $2000 \mathrm{~mm}$ ), des températures moyennes $\left(24^{\circ} \mathrm{C}\right)$. La moyenne minimale de température est de $22,5^{\circ} \mathrm{C}$ en juillet et la moyenne maximale de température de $34,6^{\circ} \mathrm{C}$ en avril. L'altitude est de $35 \mathrm{~m}$ avec un vaste réseau hydrographique constitué par la Sanaga, un des fleuves les plus importants du Cameroun. Prenant sa source dans la partie orientale du pays vers $2000 \mathrm{~m}$ d'altitude, elle draine en direction Sud-Ouest les eaux d'un vaste bassin versant, $135000 \mathrm{~km}^{2}$ à hauteur d'Edéa et se jette dans l'Atlantique au sud du port de Douala. Les sols sont ferralitiques de type sablo-argileux et latéritique de couleur brun-jaunâtre à brun vif, dérivés des roches. Matériel : Le matériel de terrain comprend : des fiches d'enquêtes, pour la collecte des informations auprès des enquêtés; des sécateurs, pour la récolte d'échantillons sur le terrain; un appareil photo numérique, pour la prise d'images ; une machette, pour déblayer le passage reculés de Ndom, Ngambe et Pouma les populations dépendent encore aujourd'hui, dans une large mesure, de la médecine traditionnelle, pour satisfaire leurs besoins en santé, malgré la présence de la médecine moderne. L'objectif général du présent travail est de contribuer à la connaissance de l'ethnomédecine de la Sanaga Maritime par une étude approfondie des recettes médicamenteuses à base des plantes. Les objectifs spécifiques sont: (1) caractériser les enquêtés, (2) déterminer les paramètres écologiques des espèces végétales identifiées, (3) recenser les recettes des plantes utilisées pour le traitement des maladies et (4) énumérer les propriétés thérapeutiques des métabolites secondaires.

broussailleux sur le terrain et récolter aussi des organes du végétal ; des papiers journaux, pour la conservation des échantillons récoltés sur le terrain; de l'alcool dilué à $70{ }^{\circ} \mathrm{C}$, pour tremper les échantillons et mieux les conserver; des presses en bois, pour presser et sécher les échantillons ; des sacs plastiques, pour le transport du matériel utilisé et des échantillons en vrac c'est-à-dire non encore sélectionnés. Tout ce matériel était nécessaire pour une bonne récolte et une meilleure conservation des échantillons obtenus sur le terrain. Les plantes sont récoltées à l'état frais dans leur biotope, en s'assurant de la période de récolte de chacune d'elle. La récolte se fait durant la floraison ou la fructification en raison de la richesse de ces plantes médicinales.

\section{Méthodologie}

Enquêtes socio-économiques: Des enquêtes ethnobotaniques basées sur les interrogations directes portant sur les usages des plantes citées dans la pharmacopée traditionnelle ont été conduites entre le mois de décembre et de mars 2016 dans les villages de Ndom, Ngambe et Pouma. L'approche des enquêtés dont l'âge a varié de]29ans, 70 ans] a été basée sur le dialogue en langues française et locale. Les personnes enquêtées devaient donner toutes les informations relatives aux plantes médicinales. Les descriptions botaniques et les différents usages des plantes médicinales rencontrées ont été complétés par des informations fournies par Letouzey, et celles recueillies à l'Herbier National du Cameroun (Letouzey, 1970). Les échantillons ont été récoltés et conservés selon les techniques et méthodes appropriées (Schnell, 1960). 


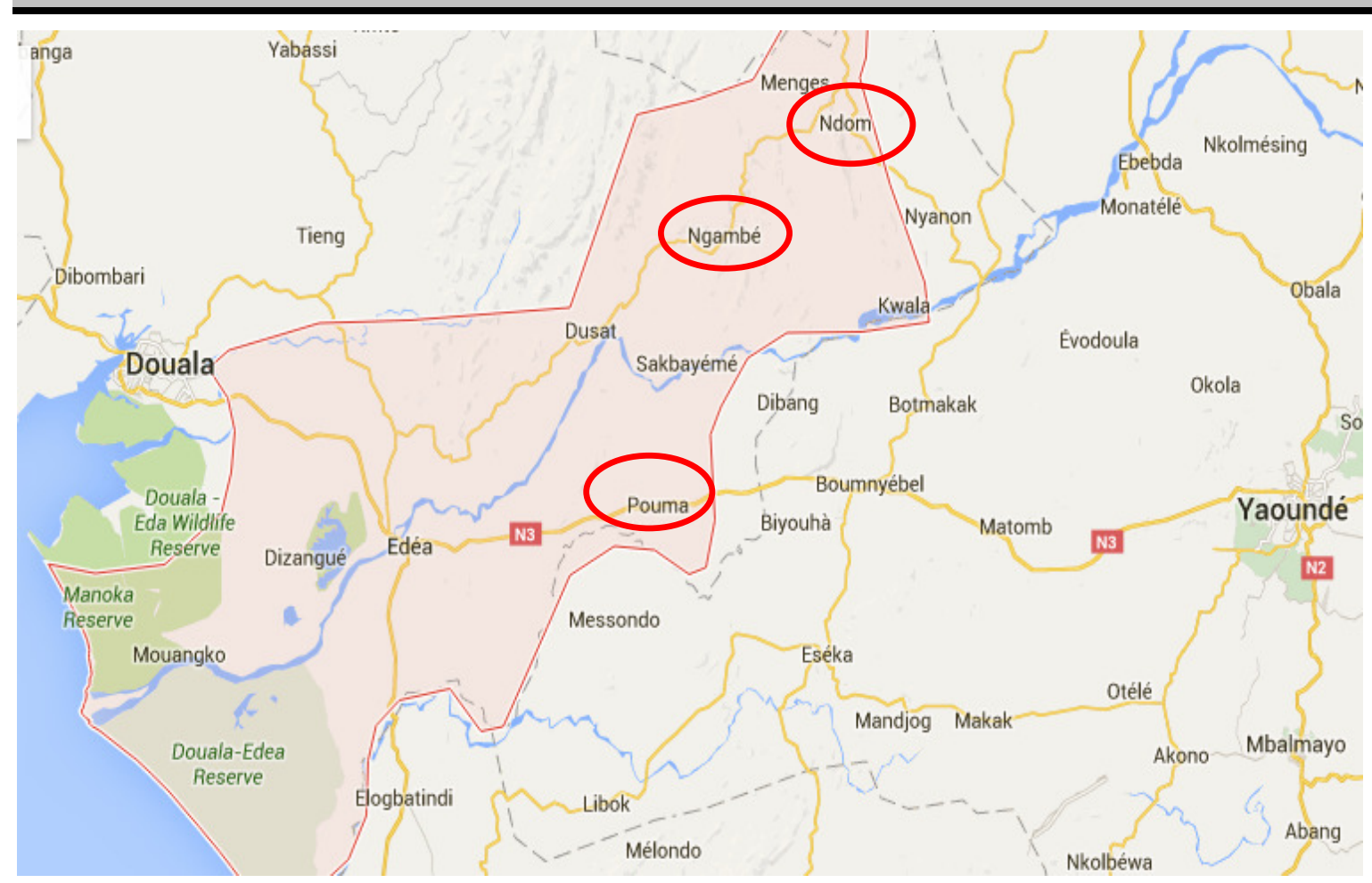

\section{: Sites d'étude}

Figure 1: Carte de localisation de la Sanaga maritime (Map data @ 2016 Google)

L'étude a été réalisée du 15 décembre 2015 au 30 mars 2016, auprès des populations rurales ayant un âge compris entre]29 ans, 70 ans].

Citations des plantes: Chaque enquêté donne les informations sur les maladies et symptômes qu'il connaît et dont il prescrit le traitement; il cite également les plantes et éléments qui entrent dans les différents traitements. L'enquêté a été appelé à citer la plante principale et les plantes associées utilisées pour le traitement d'une maladie. Les échantillons des plantes ont été récoltés et leur identification validée par les botanistes de la Faculté des Sciences de l'Université de Douala et de l'Herbier National du Cameroun, en utilisant les ouvrages des flores de l'Afrique de l'ouest et centrale ainsi que ceux du Cameroun et Gabon. Toutes les espèces nommées ont été répertoriées et identifiées.

\section{$\mathrm{FC}=\frac{\text { Nombre de citation } \mathrm{d}^{\prime} \text { une espêce }}{\text { Nombre total dienquêtés }} \times 100$}

Identification des espèces végétales utilisées comme plantes médicinales: Pour recenser les plantes, les espèces ont été reconnues dans leur milieu naturel, des prises de vue ont été effectué à l'aide d'un appareil photo numérique et la nomenclature a été donnée en langue vernaculaire ou en utilisant les noms communs. L'identification a été faite sur le terrain et au laboratoire de biologie des organismes végétaux de la Faculté des Sciences.

Description des paramètres écologiques des espèces végétales recensées : Les espèces végétales identifiées ont été décrites en utilisant les types phytogéographiques, les types morphologiques, les types de biotopes et les types de diaspores. Cette description a été rendue possible en utilisant la documentation scientifique appropriée à savoir les différents volumes de la flore du Cameroun stockée à l'Herbier National du Cameroun.

Recettes et vertus de plantes: Les recettes ont été recensées à l'aide des fiches d'enquêtes remplies à base d'informations reçues des enquêtés, soit 30 enquêtés par site étudié. Après identification, l'enquêté était amené à donner les informations concernant les recettes de plantes citées.

Détermination des propriétés thérapeutiques des métabolites secondaires: Les propriétés thérapeutiques de ces plantes ont été déterminées en utilisant la documentation en rapport avec les travaux 
relatifs au screening phytochimique, à l'étude phytochimique et à l'étude pharmacologique.

Analyse des données : Les données de terrain ont été enregistrées sur un tableur Excel, puis analysées

\section{RESULTATS}

Caractéristiques sociodémographiques des enquêtés : L'étude menée dans trois arrondissements (Ndom, Ngambe, Pouma) de la Sanaga maritime a permis d'interviewer 94 personnes. La majorité des enquêtés est constituée des hommes (55,32\%). En fonction des groupes d'âge, les adultes ont constitué la majorité des personnes enquêtées $(68,09 \%)$. La région statistiquement et traitées par le logiciel XLSTAT version 11.0.0.28480.

majoritaire est celle du Littoral. 42,55\% d'enquêtés admettent avoir reçu leur connaissances de leurs ascendants, 38,30\% l'avoir appris et $19,15 \%$ de manière empirique et héréditaire. Le pourcentage le plus élevé d'enquêtés interviewés est observé à Ngambe, soit $46,81 \%$ (Tableau 1).

Tableau 1: Caractéristiques socio démographiques des interviewés $(n=94)$

\begin{tabular}{c|c}
\hline Caractéristiques & Fréquence (\%) \\
Genre & 55,32 \\
Hommes & 44,68 \\
Femmes & \\
Age & 22,34 \\
Jeunes $\leq 29$ ans & 68,09 \\
Adultes [30-59] & 9,57 \\
3e âge [60-70] & 97 \\
Région d'origine & 1 \\
Littoral & 1 \\
Ouest & 1 \\
Est & \\
Centre & 42,55 \\
Néréditaire & 38,30 \\
Empirique & 19,15 \\
Héréditaire et empirique & \\
Répartition par village & \\
Ndom & 23,40 \\
Ngambe & 46,81 \\
Pouma & 29,79 \\
\hline
\end{tabular}

Paramètres écologiques des espèces végétales identifiées : $L$ 'inventaire floristique des villages a permis d'identifier 54 familles réparties en 92 genres et 94 espèces. Les familles majoritaires étant les Fabaceae avec $9,68 \%$ suivies des Euphorbiaceae et Asteraceae soit $6,45 \%$ chacune. Les espèces identifiées comptent 12 types de biotopes dont les plus représentés sont : les milieux cultivés $(28,85 \%)$ et forêts primaires, soit $21,15 \%$. Les plantes recensées comptent 16 types phytogéographiques, la majorité est pantropicale (24,04\%), suivie des Afro-tropicales $(20,19 \%)$ et guinéo congolaises, soit $19,23 \%$. La majorité des plantes médicinales identifiées sont des herbacées $(37,50 \%)$, suivis des arbres $(29,81 \%)$ et des arbustes soit $27,88 \%$. Les espèces inventoriées se répartissent en 7 types de diaspores avec une majorité des sarcochores $(43,27 \%)$, et sclérochores (13,46\%).

Modes de préparation, modes d'administration, recettes des plantes, maladies et symptômes traités : Le mode de préparation majoritaire utilisé pour les recettes des espèces végétales est la décoction $(27,46 \%)$, suivie de macération $(23,94 \%)$ et la friction soit $19,01 \%$. Onze modes ont été retenus dans l'administration des recettes de plantes médicinales. Les voies les plus utilisées sont: orale $(51,27 \%)$, rectale $(20,89 \%)$ et cutanée soit $12,66 \%$. L'étude 
ethnopharmacologique a permis de distinguer 80 recettes à base de plantes médicinales accompagnées de leur mode de préparation et d'administration. Le facteur de citation (Fc) calculé est compris entre $1,06 \%$ et $12,77 \%$ (Tableau 2).

Propriétés thérapeutiques des métabolites secondaires : Diverses parties des plantes identifiées sont représentées par les feuilles $(61,18 \%)$, suivies des écorces $(22,35 \%)$ et des fruits. Les métabolites secondaires sont des composés retrouvés soit dans une partie des organes végétaux, soit dans tous les organes de la plante. Leurs propriétés seraient comparables aux indications données par les populations. Les alcaloïdes et les flavonoïdes ont été spécifiques des Acanthaceae, ont des propriétés antispasmodique, antalgique, antiallergique ce qui permet de traiter les maladies et symptômes telles que la gastralgie, anémie, érythème fessier du nourrisson. Les terpènes et flavonoïdes ont été spécifiques des Asteraceae, qui sont décongestionnant, antiseptique, antalgique, antimicrobienne, antiinflammatoire, stimulant digestif et hypotenseur permettant le traitement des caries dentaires, plaies, dysenterie amibienne, sinusite, paludisme, varicelle, gastralgie, diabète, conjonctivite etc. Les terpènes et alcaloïdes ont été spécifiques des Euphorbiaceae, ont des propriétés antispasmodique, antimicrobienne et antalgique bénéfiques dans le traitement de syphilis, gastralgie et colites. Les tanins et saponines ont été majoritaires chez les Fabaceae, avec des propriétés anti diarrhéique, antiulcéreuse, antihémorragique, antiinflammatoire, cicatrisante et antalgique permettant de soigner les lombalgie, hémorroïdes, dysenterie amibienne, dysménorrhées, gastralgie, spasmes. Les alcaloïdes majoritaires des Lamiaceae, responsables des activités antispasmodique, anticancéreuse, laxative, antirhumatismale, et antalgique interviennent dans le traitement de la dysenterie amibienne, épistaxis, zona et typhoïde. Les propriétés des classes de métabolites obtenues pour les familles de plantes précédemment citées confirment les indications obtenues au cours de l'étude. 
Nga et al. J. Appl. Biosci. 2016

Inventaire et caractérisation des plantes médicinales utilisées en thérapeutique dans le département de la Sanaga Maritime : Ndom, Ngambe et Pouma

\begin{tabular}{|c|c|c|c|c|c|c|c|}
\hline Familles & $\begin{array}{l}\text { Plantes } \\
\text { principales }\end{array}$ & $\begin{array}{l}\text { Plantes } \\
\text { associées }\end{array}$ & $\begin{array}{l}\text { Organe } \\
\text { s } \\
\text { utilisés }\end{array}$ & Fc & $\begin{array}{l}\text { Maladies et symptômes } \\
\text { soignés }\end{array}$ & Mode de préparation & $\begin{array}{l}\text { Mode d'administration, } \\
\text { posologie et durée du } \\
\text { traitement }\end{array}$ \\
\hline 1.Acanthaceae & $\begin{array}{l}\text { Acanthus } \\
\text { montanus }\end{array}$ & Dacryodes edulis & Feuilles & 2,13 & Hypervolémie & $\begin{array}{l}\text { Piler et macérer dans } 2 \text { I } \\
\text { d'eau et de vin de palme } \\
\text { Décoction }\end{array}$ & $\begin{array}{l}1 \text { verre } M \text { et } S \text { jusqu'à disparition } \\
\text { de la douleur } \\
1 / 2 \text {-1 verre /j et mesurer la } \\
\text { tension }\end{array}$ \\
\hline 2. Acanthaceae & $\begin{array}{l}\text { Eremomastax } \\
\text { speciosa }\end{array}$ & $\begin{array}{l}\text { Ageratum } \\
\text { conyzoides, } \\
\text { Vernonia } \\
\text { stellulifera }\end{array}$ & Feuilles & 4,26 & $\begin{array}{l}\text { Anémie } \\
\text { Stérilité }\end{array}$ & $\begin{array}{l}\text { Decoction } \\
\text { Maceration }\end{array}$ & $\begin{array}{l}1 \text { verre } M \text { et } S \text { pendant } 1 \text { mois } \\
2 \text { poires de } 8 / j \text { pendant } 3 j, \\
\text { répéter } 2-3 \text { fois } \\
\text { Appliquer sur les parties atteintes } \\
\text { après chaque bain/1c à c } M \text { et } S\end{array}$ \\
\hline & & & & & $\begin{array}{l}\text { Erythème fessier du } \\
\text { nourisson }\end{array}$ & Friction & \\
\hline 3. Acanthaceae & $\begin{array}{l}\text { Graptophyllum } \\
\text { pictum }\end{array}$ & / & Feuilles & 1,06 & Anémie & Décoction dans 1 I d'eau & $\begin{array}{l}1 \text { verre } \mathrm{M} \text { et } \mathrm{S} \text { avec du lait } \\
\text { pendant } 3 \mathrm{j}\end{array}$ \\
\hline 4. Acanthaceae & $\begin{array}{l}\text { Hypoestes } \\
\text { aristata }\end{array}$ & $\begin{array}{l}\text { Clerodendrum cf. } \\
\text { splendens }\end{array}$ & Feuilles & 2,13 & Zona & $\begin{array}{l}\text { Ecraser et mélanger à } \\
\text { l'huile de palmiste }\end{array}$ & $\begin{array}{l}\text { Appliquer sur la partie atteinte } \\
\text { jusqu'à disparition de l'éruption }\end{array}$ \\
\hline 5. Aloeceae & Aloe vera & $\begin{array}{l}\text { Citrus lemon, } \\
\text { Capsicum } \\
\text { frutescens }\end{array}$ & Feuilles & 2,13 & $\begin{array}{l}\text { Goitre } \\
\text { Lombalgie }\end{array}$ & $\begin{array}{l}\text { Assaisonner la feuille de } \\
\text { jus de lemon, } \\
\text { Piler avec petits piments et } \\
\text { faire une décoction }\end{array}$ & $\begin{array}{l}\text { manger à volonté jusqu'à } \\
\text { diminution de l'inflammation } \\
2 \text { poires/j tous les } 2 \text { j répéter } 3 \\
\text { fois }\end{array}$ \\
\hline $\begin{array}{l}6 . \\
\text { Amaranthaceae }\end{array}$ & $\begin{array}{l}\text { Cyathula } \\
\text { prostrata }\end{array}$ & $\begin{array}{l}\text { Aframomum } \\
\text { melegueta }\end{array}$ & Feuilles & 2,13 & Diarrhée & $\begin{array}{l}\text { Ecraser avec } 9 \text { grains de } \\
\text { ndong }\end{array}$ & $\begin{array}{l}\text { Déposer au niveau du palais } \\
\text { supérieur tous les } 2 \text { jours jusqu'à } \\
\text { arrêt de la traction de la langue } \\
2 \text { gouttes/ œil une seule fois }\end{array}$ \\
\hline $\begin{array}{l}7 . \\
\text { Amaryllidaceae }\end{array}$ & $\begin{array}{l}\text { Allium cepa } \\
\text { var. } \\
\text { aggregatum }\end{array}$ & $\begin{array}{l}\text { Cola nitida, } \\
\text { Aframomum } \\
\text { melegueta }\end{array}$ & Feuilles & 1,06 & Appendicite & $\begin{array}{l}\text { Piler/ frictionner } \\
\text { Mastication }\end{array}$ & Manger à volonté pendant 1 jour \\
\hline $\begin{array}{l}8 . \\
\text { Amaryllidaceae }\end{array}$ & Allium cepa & / & Bulbe & 1,06 & $\begin{array}{l}\text { Toux } \\
\text { Marche difficile de l'enfant } \\
\text { Hernie ombilicale }\end{array}$ & $\begin{array}{l}\text { Décoction + sucre } \\
\text { Macération }\end{array}$ & $\begin{array}{l}1 \text { càs } M \text { et } S \text { pendant } 5 \text { jours } \\
\text { Purger pendant } 3 \mathrm{~J} \text {, } \\
\text { Inciser les membres et appliquer } \\
\text { si échec de la purge, } \\
1 \text { purge(Ad), } 1 / 2 \text { purge(enfant)/j } \\
\text { pendant } 2 \mathrm{j}\end{array}$ \\
\hline
\end{tabular}


9

Anacardiaceae

10.

Anacardiaceae

11. Annonaceae

12.

Apocynaceae

13. Arécaceae

14. Asteraceae

15. Asteraceae

\section{Anthrocaryon} micraster

\section{Mangifera}

indica

\section{Annona} muricata

Alstonia boonei

Elaeis

guineensis

Acmella caulirhiza

Ageratum conyzoides

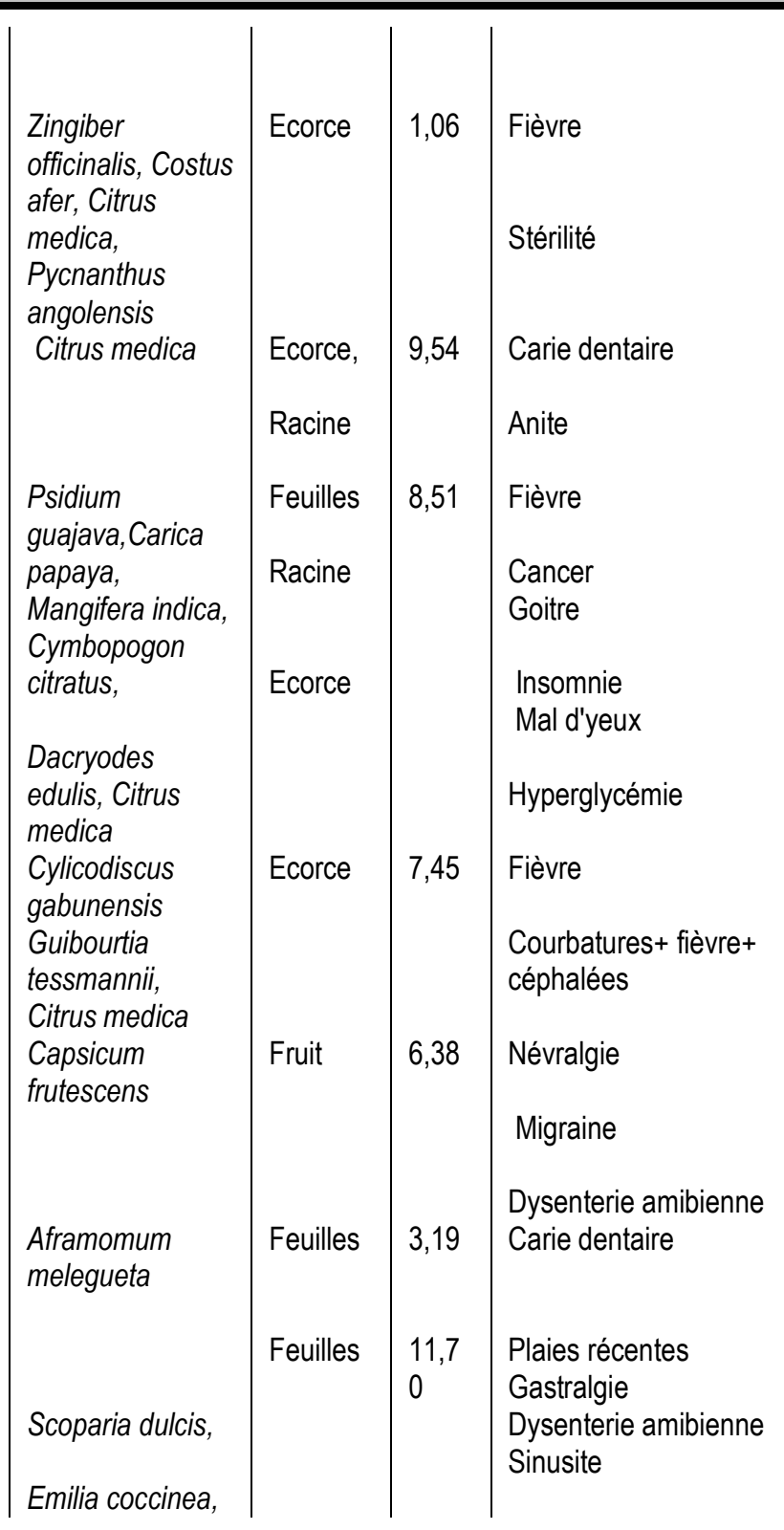

Ecraser et faire une

décoction

Piler, écraser et faire une

décoction; Expression de

Costus afer, ajouter le

citron (10citrons pour 5I)

Décoction

Macération dans le jus de citron

Décoction

Décoction

Infusion

Ecraser et macérer

Décoction

Décoction/ macération

Piler et macérer pendant

$12 \mathrm{~h}$

Mâcher et appliquer

Broyage et macération

avec du sel

Ecraser+9grains de Ndong

ajouter quelques gouttes

d'alcool

Macération

Friction

Friction

Expression et faire un 1verre tiède $\mathrm{M}$ et $\mathrm{S}$ pendant 1semaine

2 poires/j tous les 2 j répéter 3 fois et garder le reste

bain de bouche à chaque douleur

Bain de siège 3 fois/j pendant 2

semaines et $1,5 \mathrm{I} M, \mathrm{M}, \mathrm{S}$

1 verre $M, M, S$ terminer $1,5 \mathrm{~L}$

1verre Met S pendant 21j (3j

goitre)

1 tasse/J

2gouttes/œil pendant 2j

1verre/j

$1-2$ càc de sève/j pendant 3jours 1 verre Met $S$ pendant $3 j-$ 1semaine

application unique sous le soleil Appliquer

1 fois/j pendant 2j

1 verre $M$ et $S$ pendant 1 semaine

Déposer sur la dent pendant 15 min et observer la réaction pendant $2 \mathrm{j}$

Déposer

1 verre $M, M, S$ 1verre/j prise

unique

3gouttes M, M, S pendant 7j

(couché) 


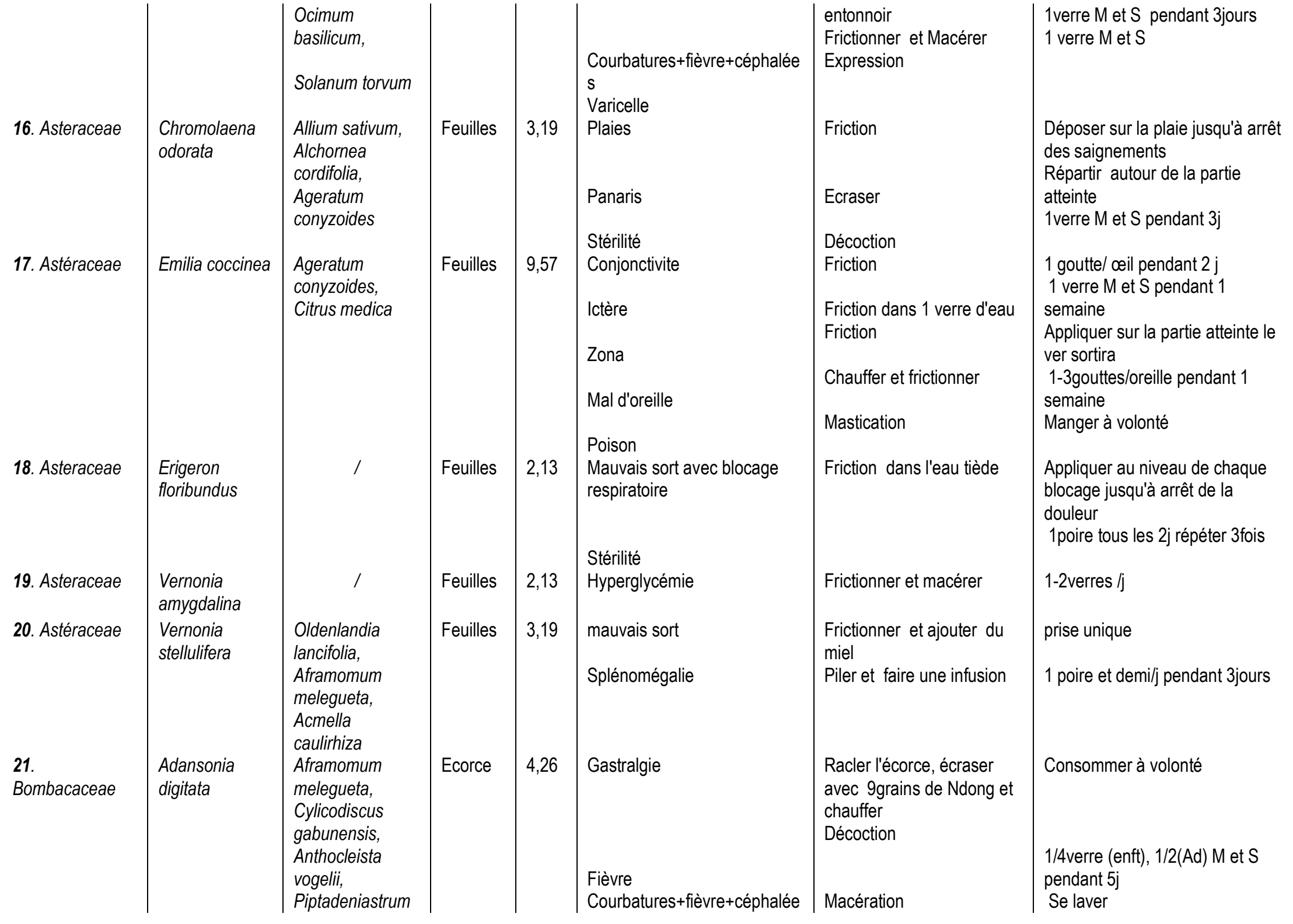

1 verre $\mathrm{M}$ et $\mathrm{S}$ pendant 3jours

entonnoir

Frictionner et Macérer

1 verre $M$ et $S$

basilicum,

Solanum torvum

Ageratum

Expression

Frictio

Ecraser

Friction dans 1 verre d'eau

Mastication

Friction dans l'eau tiède

Frictionner et macére

Frictionner et ajouter $d$

Piler et faire une infusion

Racler l'écorce, écrase

Macération
Se laver 


\begin{tabular}{|c|c|c|c|c|c|c|c|}
\hline & & $\begin{array}{l}\text { africanum, } \\
\text { Entandrophragm } \\
\text { a cylindricum, } \\
\text { Entandrophragm } \\
\text { a utile }\end{array}$ & & & $\begin{array}{l}\text { S } \\
\text { Purification } \\
\text { Spasmes }\end{array}$ & Décoction & 1 poire le soir avant le coucher \\
\hline 22. Burseraceae & $\begin{array}{l}\text { Dacryodes } \\
\text { edulis }\end{array}$ & $\begin{array}{l}\text { Mangifera indica, } \\
\text { Cymbopogon } \\
\text { citratus, Persea } \\
\text { americana, } \\
\text { Citrus medica, } \\
\text { Carica papaya }\end{array}$ & $\begin{array}{l}\text { Feuilles, } \\
\text { écorce }\end{array}$ & 4,26 & $\begin{array}{l}\text { Douleurs post partum, } \\
\text { poison } \\
\text { Fièvre }\end{array}$ & Piler et faire une décoction & $\begin{array}{l}\text { massage } 1 \text { fois/j jusqu'à disparition } \\
\text { des douleurs } \\
\text { Boire à volonté jusqu'à } \\
\text { rétablissement; } \\
1 \text { verre } \mathrm{M}, \mathrm{M}, \mathrm{S} \text { pendant } 5 \mathrm{j}\end{array}$ \\
\hline $\begin{array}{l}23 . \\
\text { Capparidaceae }\end{array}$ & Cleome ciliata & $\begin{array}{l}\text { Aframomum } \\
\text { exscapum }\end{array}$ & $\begin{array}{l}\text { Feuilles } \\
\text { et tiges }\end{array}$ & 1,06 & Douleurs ombilicales & $\begin{array}{l}\text { Piler avec } 1 \text { quartier de } \\
\text { Mbongo, tamiser et laisser } \\
\text { macérer }\end{array}$ & 1 petite poire $/ \mathrm{j}$ pendant 3 jours \\
\hline 24. Caricaceae & Carica papaya & $\begin{array}{l}\text { Allium sativum, } \\
\text { Zea mays, Citrus } \\
\text { medica, Citrus } \\
\text { lemon }\end{array}$ & $\begin{array}{l}\text { Feuilles, } \\
\text { pépins }\end{array}$ & $\begin{array}{l}10,6 \\
4\end{array}$ & $\begin{array}{l}\text { Fibromes } \\
\text { Helminthiase } \\
\text { Courbatures +fièvre+ } \\
\text { céphalées }\end{array}$ & $\begin{array}{l}\text { Sécher, écraser et faire } \\
\text { une infusion } \\
\text { Décoction } \\
\text { Décoction avec le miel } \\
\text { Infusion avec le miel et le } \\
\text { lait }\end{array}$ & $\begin{array}{l}1 \text { poire } / \mathrm{j} \text { tous les } 3 \text { jours } \\
\text { 1verre } 3-5 \text { fois/j } \\
\text { 1verre } M, M, S \text { pendant } 1-2 \\
\text { semaine } \\
1 / 2 \text { verre } M \text { et } S \text { pendant } 1 \\
\text { semaine }\end{array}$ \\
\hline 25. Caricaceae & $\begin{array}{l}\text { Carica papaya } \\
\text { var. solo }\end{array}$ & / & $\begin{array}{l}\text { Fruit, } \\
\text { pépins }\end{array}$ & 1,06 & Fièvre & $\begin{array}{l}\text { Piler le fruit, écraser les } \\
\text { pépins et mélanger }\end{array}$ & $\begin{array}{l}\text { Consommer toute la potion par } \\
\text { jour pendant } 5 \text { jours }\end{array}$ \\
\hline 26. Clusiaceae & $\begin{array}{l}\text { Mammea } \\
\text { africana }\end{array}$ & $\begin{array}{l}\text { Cylicodiscus } \\
\text { gabunensis }\end{array}$ & Ecorce & 2,13 & Chlamydia & Décoction & $\begin{array}{l}2 \text { poires de } 8 \text { ou de } 10 / j \text { pendant } \\
\text { 3j par semaine répéter pendant } 3 \\
\text { semaines }\end{array}$ \\
\hline 27. Connaraceae & $\begin{array}{l}\text { Cnestis } \\
\text { ferruginea }\end{array}$ & / & Fruit & 1,06 & Gingivite & Friction & $\begin{array}{l}\text { Frotter } 1 \text { fruit à chaque douleur } \\
\text { sur la gencive jusqu'à disparition } \\
\text { de l'inflammation }\end{array}$ \\
\hline $\begin{array}{l}28 . \\
\text { Convolvulaceae }\end{array}$ & Ipomea batatas & $\begin{array}{l}\text { Aframomum } \\
\text { melegueta }\end{array}$ & Feuilles & 1,06 & Panaris & $\begin{array}{l}\text { Ecraser, empaqueter et } \\
\text { chauffer près du feu }\end{array}$ & $\begin{array}{l}\text { Masser une seule fois/ déposer à } \\
\text { l'endroit } 1 \mathrm{j}\end{array}$ \\
\hline 29. Costaceae & Costus afer & / & Rhizome & 7,45 & $\begin{array}{l}\text { Varicelle } \\
\text { Hyperglycémie } \\
\text { Ictère }\end{array}$ & $\begin{array}{l}\text { Sucer } \\
\text { Expression } \\
\text { Expression et chauffer }\end{array}$ & $\begin{array}{l}\text { sucer jusqu'à disparition de } \\
\text { l'éruption } \\
1 / 2 \text { verre/j pendant } 2 \text { mois } \\
1 \text { verre avec } 1 \text { morceau de sucre } \\
\text { /j pendant } 1 \text { semaine; } \\
\text { 2gouttes/narine } \\
\text { 1goutte/œil M et S pdt 1semaine }\end{array}$ \\
\hline
\end{tabular}




\section{}

30. Crassulaceae

Kalanchoe crenata

31.

Cucurbitaceae

32. Davalliaceae

33.

Euphorbiaceae

34.

Euphorbiaceae

35.

Euphorbiaceae

36.

Euphorbiaceae

37.

Euphorbiaceae

38. Fabaceae

39. Fabaceae

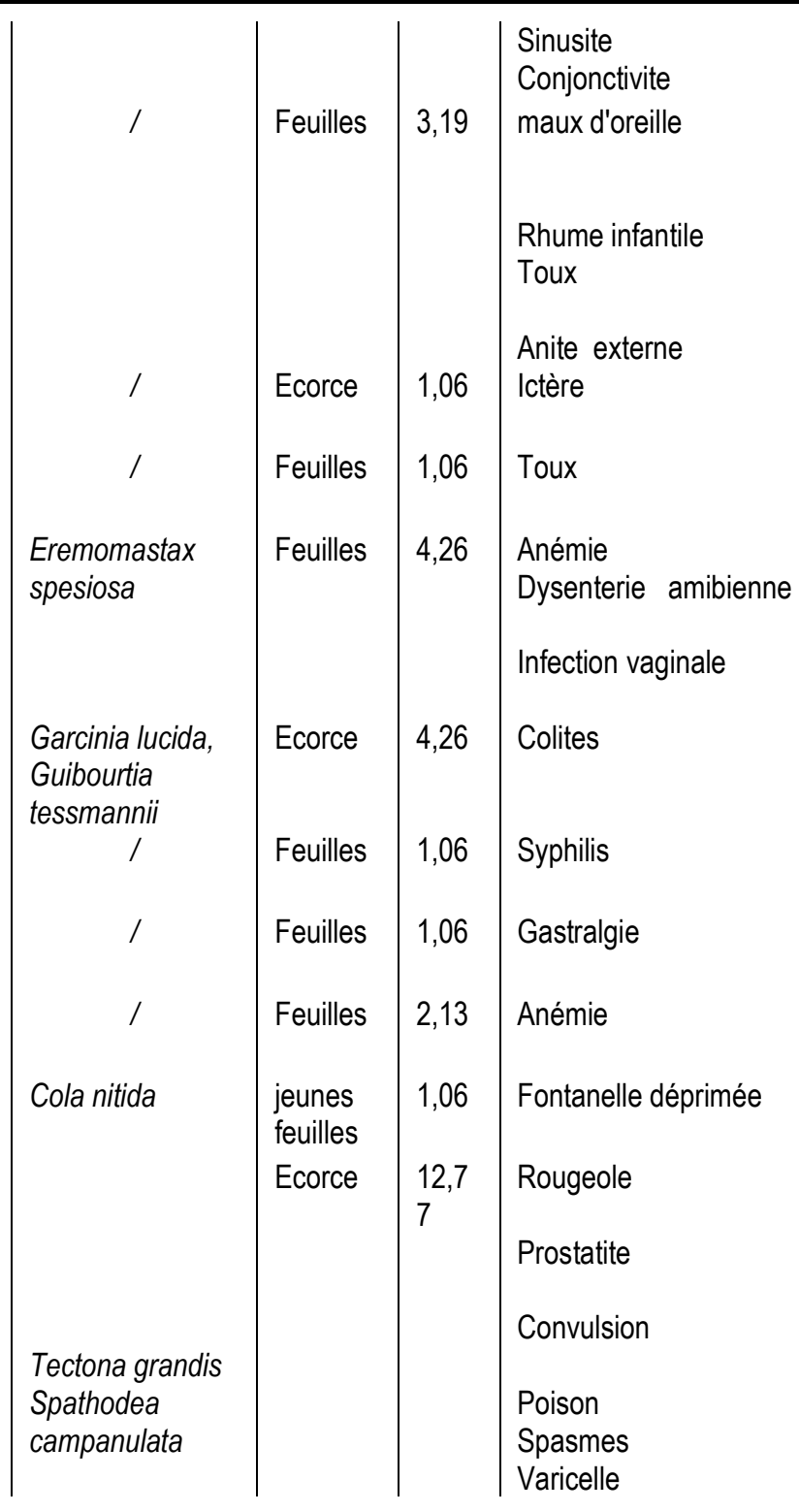

Tirer les fibres

Chauffer et ajouter I'huile

de palmiste

Chauffer et frictionner

Chauffer et frictionner

Chauffer et frictionner

Macération

Piler, macérer dans 2l

d'eau et tamiser

Macération

Mastication

Friction et enrouler

Sécher et écraser

Frictionner et tamiser

Friction et macération

Expression et ajouter du

lait

Ecraser et mélanger au lait maternel

Macération avec le vin de

palme

Décoction

Macération

Décoction

Macération
1 goutte/oreille jusqu'à disparition de la douleur

2 gouttes/narine $M$ et $S$ pendant $3 j$

1 càc/j pendant $3 \mathrm{j}$

Approcher de l'anus une seule fois

1 verre $\mathrm{M}$ et $\mathrm{S}$ pendant $2 \mathrm{j}$

1 verre $\mathrm{M}$ et $\mathrm{S}$ pendant 3-5j

1verre M, M, S

1-2 feuilles prise unique

Introduire dans le vagin au

coucher pendant 1 semaine

1 càc de poudre /j

1 verre $M$ et $S$ pendant 1 semaine

si aigue

1 verre $M$ et $S$

1 tasse /j pendant une semaine

2-3gouttes/j jusqu'à disparition

des battements

se purger et boire

Se purger M et S pendant 4-

5jours

Laver l'enfant 2fois /j

1 verre $\mathrm{M}, \mathrm{M}, \mathrm{S}$

1verre/j pendant 3j

1verre/j pendant 1semaine 


\begin{tabular}{|c|c|c|c|c|c|c|c|}
\hline 40. Fabaceae & $\begin{array}{l}\text { Desmodium } \\
\text { adscendens }\end{array}$ & Elaeis guineensis & Feuilles & 6,38 & Dysenterie amibienne & Laver et mâcher & 1 fois par jour pendant 3 jours \\
\hline 41. Fabaceae & $\begin{array}{l}\text { Erythrophleum } \\
\text { ivorense }\end{array}$ & $\begin{array}{l}\text { Annickia } \\
\text { chlorantha, } \\
\text { Cylicodiscus } \\
\text { gabunensis }\end{array}$ & Ecorce & 1,06 & Convulsion infantile & $\begin{array}{l}\text { Sécher, racler et obtenir } \\
\text { une poudre; Piler et laisser } \\
\text { macérer au soleil }\end{array}$ & Laver l'enfant 2 fois / $\mathrm{j}$ \\
\hline 42. Fabaceae & $\begin{array}{l}\text { Guibourtia } \\
\text { tessmannii }\end{array}$ & / & Ecorce & 6,38 & $\begin{array}{l}\text { Dysménorrhées } \\
\text { Envoûtement }\end{array}$ & Décoction & $\begin{array}{l}\text { 3petites poires (enfant), } 2 \\
\text { grandes poires }(\mathrm{Ad}) / \mathrm{j} \text { tous les } 4 \mathrm{j} \\
\text { à répéter } 3 \text { fois } \\
\text { 1 verre matin après le repas } \\
\text { pendant } 1-3 \text { mois }\end{array}$ \\
\hline 43. Fabaceae & Mimosa pudica & / & Feuilles & 1,06 & Dysenterie amibienne & $\begin{array}{l}\text { Broyage } \\
\text { découper en petits } \\
\text { morceaux, faire une } \\
\text { décoction dans 3l d'eau } \\
\text { +sel gemme }\end{array}$ & $\begin{array}{l}1,5 \mathrm{l} \text { pour } 2 \mathrm{j} \mathrm{M}, \mathrm{M}, \mathrm{S} \text { un traitement } \\
=3 \mathrm{l}\end{array}$ \\
\hline 44. Fabaceae & Senna alata & Elaeis guineensis & Feuilles & 4,26 & $\begin{array}{l}\text { Dartre } \\
\text { Fièvre jaune } \\
\text { Erythème fessier } \\
\text { Teigne }\end{array}$ & $\begin{array}{l}\text { Ecraser avec sel gemme } \\
\text { et huile de palmiste } \\
\text { Décoction avec le sel } \\
\text { gemme } \\
\text { Macération } \\
\text { Friction }\end{array}$ & $\begin{array}{l}\text { S'oindre jusqu'à disparition des } \\
\text { tâches } \\
\text { 1,5l/J pendant } 2 \text { j } \\
\text { 1purge/j jusqu'à guérison } \\
\text { Appliquer 2fois/j sur les parties } \\
\text { affectées }\end{array}$ \\
\hline 45. Fabaceae & $\begin{array}{l}\text { Piptadeniastru } \\
\text { m africanum }\end{array}$ & / & Ecorce & 2,13 & Lombalgie & Décoction & 1 poire/j pendant 1 semaine \\
\hline 46. Fabaceae & $\begin{array}{l}\text { Tetrapleura } \\
\text { tetraptera }\end{array}$ & $\begin{array}{l}\text { Aframomum } \\
\text { exscapum }\end{array}$ & Fruit & 1,06 & Anite & $\begin{array}{l}\text { Ecraser sur une pierre } \\
\text { propre }\end{array}$ & $\begin{array}{l}\text { Introduire un morceau de pate } \\
\text { après chaque selle }\end{array}$ \\
\hline 47. Gnetaceae & $\begin{array}{l}\text { Gnetum } \\
\text { africanum }\end{array}$ & / & Feuilles & 1,06 & Anémie & Piler, macérer et tamiser & $1 / 2$ verre/j pendant $2-3 j$ \\
\hline 48. Lamiaceae & $\begin{array}{l}\text { Clerodendrum } \\
\text { cf. splendens }\end{array}$ & / & Feuilles & 1,06 & Zona & Friction dans 5 I d'eau & Boire à volonté pendant $2 \mathrm{j}$ et demi \\
\hline 49. Lamiaceae & Ocimum canum & Citrus medica & Feuilles & 1,06 & Fièvre & Frictionner et macérer & 1,5L /jour pendant $5 \mathrm{j}$ \\
\hline 50. Lamiaceae & $\begin{array}{l}\text { Ocimum } \\
\text { basilicum }\end{array}$ & / & Feuilles & 3,19 & Epistaxis & $\begin{array}{l}\text { Frictionner pour obtenir } \\
\text { une pate }\end{array}$ & $\begin{array}{l}\text { introduire dans les narines et } \\
\text { aspirer; } 2 \text { gouttes/narine prise } \\
\text { unique, poursuivre si l'écoulement } \\
\text { continue }\end{array}$ \\
\hline
\end{tabular}


Nga et al. J. Appl. Biosci. 2016

Inventaire et caractérisation des plantes médicinales utilisées en thérapeutique dans le département de la Sanaga Maritime : Ndom, Ngambe et Pouma

\begin{tabular}{|c|c|c|c|c|c|c|c|}
\hline 51.Lamiaceae & $\begin{array}{l}\text { Tectona } \\
\text { grandis }\end{array}$ & $\begin{array}{l}\text { Anthocleista } \\
\text { vogelii, } \\
\text { Mangifera indica, } \\
\text { Guibourtia } \\
\text { tessmannii }\end{array}$ & Ecorce & 4,26 & $\begin{array}{l}\text { Dysenterie amibienne } \\
\text { Splénomégalie }\end{array}$ & $\begin{array}{l}\text { Macération } \\
\text { Décoction } \\
\text { Décoction + vin de palme }\end{array}$ & $\begin{array}{l}1 \text { verre } M \text { et } S \text { pendant } 5-6 \mathrm{j} \\
1 / 2 \text { purge/j tous les } 2 \text { j pendant } 1 \\
\text { semaine; } 2 \text { poires/j pendant } \\
\text { 5jours répéter } 3 \text { fois après } 2 \\
\text { semaines }\end{array}$ \\
\hline 52. Lauraceae & $\begin{array}{l}\text { Persea } \\
\text { americana }\end{array}$ & / & Feuilles & 5,32 & $\begin{array}{l}\text { Hyperglycémie } \\
\text { Ictère }\end{array}$ & Décoction & $\begin{array}{l}\text { 1verre M, M, S et mesurer sa } \\
\text { glycémie; } 1 \text { verre/j jusqu'à } \\
\text { guérison }\end{array}$ \\
\hline $\begin{array}{l}53 . \\
\text { Lecythidaceae }\end{array}$ & $\begin{array}{l}\text { Petersianthus } \\
\text { macrocarpus }\end{array}$ & $\begin{array}{l}\text { Citrus medica, } \\
\text { Heisteria sp. } \\
\text { Ocimum sp. }\end{array}$ & $\begin{array}{l}\text { Ecorce } \\
\text { Feuilles }\end{array}$ & 1,06 & $\begin{array}{l}\text { Panaris } \\
\text { Splénomégalie }\end{array}$ & $\begin{array}{l}\text { Ecraser la composition, } \\
\text { chauffer le paquet }\end{array}$ & $\begin{array}{l}\text { massage une fois avec la } \\
\text { combinaison, } 2 \text { fois sans plantes } \\
\text { associées }\end{array}$ \\
\hline 54. Loganiaceae & $\begin{array}{l}\text { Anthocleista } \\
\text { vogelii }\end{array}$ & $\begin{array}{l}\text { Aframomum } \\
\text { exscapum }\end{array}$ & Ecorce & 5,32 & Chlamydia & Décoction & 1 poire /j pendant $9 \mathrm{j}$ \\
\hline 55. Malvaceae & $\begin{array}{l}\text { Abelmoschus } \\
\text { esculentus }\end{array}$ & / & Fruit & 1,06 & Hyperglycémie & $\begin{array}{l}\text { Ecraser, macérer et } \\
\text { tamiser }\end{array}$ & $\begin{array}{l}1 \text { verre } \mathrm{M} \text { et } \mathrm{S} \text { et mesurer la } \\
\text { glycémie }\end{array}$ \\
\hline 56. Malvaceae & $\begin{array}{l}\text { Hibiscus } \\
\text { sabdariffa }\end{array}$ & Allium sativum & Feuilles & 1,06 & Fièvre & $\begin{array}{l}\text { Ecraser le charbon } \\
\text { macérer et boire, } \\
\text { macération d'hibiscus +ail } \\
\text { écrasé dans } 11 \text { d'eau }\end{array}$ & 1I pour 3j pendant 3 semaines \\
\hline $\begin{array}{l}57 . \\
\text { Melastomatacea } \\
e\end{array}$ & $\begin{array}{l}\text { Dissotis } \\
\text { rotundifolia }\end{array}$ & $\begin{array}{l}\text { Diodia cf. } \\
\text { scandens. } \\
\text { Desmodium } \\
\text { adscendens, } \\
\text { Costus afer, } \\
\text { Tetracera sp. } \\
\text { Aframomum } \\
\text { melegueta }\end{array}$ & Feuilles & 2,13 & $\begin{array}{l}\text { Dysenterie amibienne } \\
\text { Splénomégalie }\end{array}$ & $\begin{array}{l}\text { Ecraser avec le sel } \\
\text { Laver et écraser, } \\
\text { Ecraser et macérer }\end{array}$ & $\begin{array}{l}\text { Manger à volonté jusqu'à } \\
\text { disparition de la douleur } \\
\text { disposer sur 9doigts et manger } \\
\text { M, M, S pendant 1-3j; 2poires/j } \\
\text { tous 2j répéter 3fois }\end{array}$ \\
\hline 58. Moraceae & $\begin{array}{l}\text { Ficus } \\
\text { exasperata }\end{array}$ & $\begin{array}{l}\text { Desmodium } \\
\text { adscendens }\end{array}$ & Feuilles & 4,26 & $\begin{array}{l}\text { Panaris } \\
\text { Sinusite }\end{array}$ & $\begin{array}{l}\text { Ecraser avec le sel et 2-3 } \\
\text { gouttes de pétrole } \\
\text { Friction dans l'eau } \\
\text { Sécher et écraser } \\
\text { Macération }\end{array}$ & $\begin{array}{l}\text { Attacher tous les } 2 \mathrm{j} \\
\text { 2gouttes/narine } M \text { et } S \text { pendant } 2 \\
\text { semaines et 2aspiration de } \\
\text { poudre/ pendant } 4 \mathrm{j} \\
1-2 \text { poires/ } \mathrm{j} \text { espacé d'1j à répéter } \\
5 \text { fois }\end{array}$ \\
\hline 59. Moraceae & $\begin{array}{l}\text { Musanga } \\
\text { cecropioides }\end{array}$ & / & $\begin{array}{l}\text { Ecorce } \\
\text { Feuilles }\end{array}$ & 2,13 & $\begin{array}{l}\text { Splénomégalie } \\
\text { Lombalgie } \\
\text { Splénomégalie }\end{array}$ & Décoction & $\begin{array}{l}\text { Se faire masser sans garder le } \\
\text { reste } \\
1 \text { poire/semaine pendant } 3\end{array}$ \\
\hline
\end{tabular}




60. Musaceae

\section{Musa} paradisiaca

61.

Myristicaceae

62. Myrtaceae

63.

Passifloraceae

64. Poaceae

65. Poaceae

66.

Polypodiaceae

67. Rubiaceae

\begin{tabular}{|c|c|c|c|}
\hline $\begin{array}{l}\text { Citrus medica, } \\
\text { Croton } \\
\text { olingadrus, } \\
\text { Mangifera indica, } \\
\text { Cymbopogon } \\
\text { citratus }\end{array}$ & $\begin{array}{l}\text { Feuilles } \\
\text { jaunes } \\
\text { Ecorce }\end{array}$ & 3,19 & $\begin{array}{l}\text { Dermite } \\
\text { Fièvre } \\
\text { Splénomégalie }\end{array}$ \\
\hline & Feuilles & 5,32 & $\begin{array}{l}\text { Dysenterie } \\
\text { amibienne } \\
\text { Fièvre } \\
\text { Dysenterie amibienne } \\
\text { Spasmes }\end{array}$ \\
\hline / & Sève & 1,06 & Splénomégalie \\
\hline $\begin{array}{l}\text { Citrus medica, } \\
\text { Alstonia boonei }\end{array}$ & Feuilles & 1,06 & Toux \\
\hline 1 & Feuilles & 1,06 & Conjonctivite \\
\hline Allium cepa & Feuilles & 1,06 & Palpitations cardiaques \\
\hline $\begin{array}{l}\text { Hypoestes } \\
\text { aristata }\end{array}$ & Ecorce & 2,13 & Splénomégalie \\
\hline $\begin{array}{l}\text { Alstonia boonei, } \\
\text { Psidium guajava, } \\
\text { Annona } \\
\text { muricata, Carica } \\
\text { papaya }\end{array}$ & & & Fièvre \\
\hline
\end{tabular}

\section{semaines}

Couper le tronc et recueillir le liquide Décoction

Piler, faire une décoction

pendant 30min

Recueillir le liquide en

coupant l'écorce de l'arbre

Décoction

Mastication

Ecraser et ajouter l'huile de palmiste

Décoction

Friction

Brûler et obtenir une

cendre

Macération

Ecraser et faire une décoction
2 càs à 1 verre en prise unique;

Appliquer sur les parties irritées

1 grand verre $M$ et $S$ pendant

5 jours et bain de vapeur pendant 5 min

se purger le décocté tiède 2 fois/ pendant $3 \mathrm{j}$

2 càs/j en une prise sinon

constipation

1 verre $M, M, S$ durée indéterminée,

1 verre $\mathrm{M}$ et $\mathrm{S}$ pendant 2-3j

Manger les jeunes feuilles

1 càc/ j pendant $3 \mathrm{j}$

$1 / 2$ verre $M$ et $S$ pendant $4 j$

1 goutte/ œil M, M, S pendant 3j

Appliquer sur la poitrine pendant 45 minutes

1 poire (enfant), 2poires (Adulte)

tous les $2 \mathrm{j}$ répéter 3 fois

1 verre $M, M$ et $S$ jusqu'à guérison 


\begin{tabular}{|c|c|c|c|c|c|c|c|}
\hline 68. Rubiaceae & Ixora sp. & $\begin{array}{l}\text { Carica papaya, } \\
\text { Cymbopogon } \\
\text { citratus }\end{array}$ & Feuilles & 1,06 & Fièvre & Décoction & 1 verre $\mathrm{M}$ et $\mathrm{S}$ pendant $5 \mathrm{j}$ \\
\hline 69. Rubiaceae & $\begin{array}{l}\text { Oldenlandia } \\
\text { lancifolia }\end{array}$ & $\begin{array}{l}\text { Aframomum } \\
\text { melegueta, } \\
\text { Acmella } \\
\text { caulirhiza, } \\
\text { Solanum torvum, } \\
\text { Scoparia dulcis } \\
\text { Lavigeria } \\
\text { macrocarpa }\end{array}$ & Feuilles & 4,26 & $\begin{array}{l}\text { Bronchite } \\
\text { Mauvais sort } \\
\text { Morsure de serpent }\end{array}$ & $\begin{array}{l}\text { Bruler et obtenir une } \\
\text { poudre }\end{array}$ & $\begin{array}{l}\text { Inciser, appliquer sur les parties } \\
\text { scarifiées et faire manger à } \\
\text { l'enfant } \mathrm{M} \text { et } \mathrm{S} \text { pendant } 2 \mathrm{j} \\
\text { Inciser et appliquer à quelques } \\
\mathrm{cm} \text { de la morsure } 1 \text { fois et } 3 \text { mois } \\
\text { plus tard }\end{array}$ \\
\hline 70. Rutaceae & $\begin{array}{l}\text { Clausena } \\
\text { anisata }\end{array}$ & I & Ecorce & 1,06 & Urétrite/ salpingite & $\begin{array}{l}\text { Piler et macérer avec du } \\
\text { vin de palme }\end{array}$ & 1 verre $\mathrm{M}$ et $\mathrm{S}$ \\
\hline 71. Sapindaceae & $\begin{array}{l}\text { Allophylus } \\
\text { africanus }\end{array}$ & / & Feuilles & 1,06 & Céphalées & Friction & Humer à volonté \\
\hline $\begin{array}{l}72 . \\
\text { Scrophulariacea }\end{array}$ & Scoparia dulcis & Pistache & Feuilles & 4,26 & Abcès & Ecraser avec un peu d'eau & $\begin{array}{l}\text { Poser sur l'abcès recouvrir d'un } \\
\text { nylon et bander pendant } 3 \mathrm{j}\end{array}$ \\
\hline e & & & & & Plaies incurables & & $\begin{array}{l}\text { Laver la plaie à l'eau tiède salée, } \\
\text { déposer la pâte } 1 \text { fois/j jusqu'à } \\
\text { guérison }\end{array}$ \\
\hline 73. Solanaceae & $\begin{array}{l}\text { Capsicum } \\
\text { frutescens }\end{array}$ & / & $\begin{array}{l}\text { Feuilles } \\
\text { Fruit }\end{array}$ & 3,19 & Erythème fessier & Macération & 1 poire par jour pendant $2-3 \mathrm{j}$ \\
\hline 74. Solanaceae & $\begin{array}{l}\text { Solanum } \\
\text { incanum }\end{array}$ & / & Fruit & 2,13 & Anite & $\begin{array}{l}\text { chauffer au centre du foyer } \\
\text { à bois } \\
\text { Couper en } 2\end{array}$ & $\begin{array}{l}\text { presser et sucer plusieurs fois } \\
\text { jusqu'à arrêt du hoquet } \\
\text { Déposer sur l'anus et enfiler les } \\
\text { sous-vêtements au coucher } \\
\text { pendant } 1 \text { semaine }\end{array}$ \\
\hline 75. Solanaceae & $\begin{array}{l}\text { Solanum } \\
\text { torvum }\end{array}$ & / & $\begin{array}{l}\text { Feuilles, } \\
\text { fruit }\end{array}$ & 5,32 & $\begin{array}{l}\text { Zona } \\
\text { Poison } \\
\text { Plaies }\end{array}$ & $\begin{array}{l}\text { Mastication } \\
\text { Friction et macération }\end{array}$ & $\begin{array}{l}\text { Manger } 9 \text { fruits et une feuille prise } \\
\text { unique } \\
\text { Boire } 1 / 2 \text { verre/j Attacher dans la } \\
\text { nuit et détacher le matin }\end{array}$ \\
\hline $\begin{array}{l}76 . \\
\text { Sterculiaceae }\end{array}$ & Cola nitida & Citrus medica & Fruit & 1,06 & Toux & Mastication & $\begin{array}{l}1 \text { kola/j en suçant simultanément } \\
\text { du citron jusqu'à arrêt de la toux } \\
\text { Inciser et oindre du sel puis } \\
\text { pulvériser la kola avec la bouche }\end{array}$ \\
\hline 77. Urticaceae & $\begin{array}{l}\text { Urera cf. } \\
\text { cordifolia }\end{array}$ & $\begin{array}{l}\text { Pycnathus } \\
\text { angolensis, } \\
\text { Dioscorea sp. }\end{array}$ & Feuilles & 2,13 & $\begin{array}{l}\text { Splénomégalie } \\
\text { Splénomégalie }\end{array}$ & Piler et faire une décoction & 1 poire M et S pendant 3j \\
\hline
\end{tabular}


Nga et al. J. Appl. Biosci. 2016

Inventaire et caractérisation des plantes médicinales utilisées en thérapeutique dans le département de la Sanaga Maritime : Ndom, Ngambe et Pouma

\begin{tabular}{|c|c|c|c|c|c|c|c|}
\hline 78. Urticaceae & $\begin{array}{l}\text { Urera } \\
\text { gabonensis }\end{array}$ & $\begin{array}{l}\text { Hallea stipulosa, } \\
\text { Cylicodiscus } \\
\text { gabunensis, } \\
\text { Guibourtia } \\
\text { tesmannii }\end{array}$ & Feuilles & 1,06 & Prostatite & Piler et faire une décoction & 1poire M et S pendant 4-5j \\
\hline 79. Verbenaceae & $\begin{array}{l}\text { Lantana } \\
\text { camara }\end{array}$ & / & Feuilles & 1,06 & Otalgies & $\begin{array}{l}\text { Frictionner, faire un } \\
\text { entonnoir et chauffer près } \\
\text { du feu }\end{array}$ & $\begin{array}{l}2 \text { gouttes } M \text { et } S \text { jusqu'à } \\
\text { disparition de la douleur }\end{array}$ \\
\hline $\begin{array}{l}80 . \\
\text { Zingiberaceae }\end{array}$ & $\begin{array}{l}\text { Aframomum } \\
\text { exscapum }\end{array}$ & $\begin{array}{l}\text { Tetrapleura } \\
\text { tetraptera, } \\
\text { Zingiber } \\
\text { officinalis, } \\
\text { Cleome ciliata }\end{array}$ & Grains & 2,13 & $\begin{array}{l}\text { Anite } \\
\text { Douleur ombilicale infantile }\end{array}$ & $\begin{array}{l}\text { Ecraser et mélanger dans } \\
1 / 2 \text { verre d'eau } \\
\text { Macérer et tamiser }\end{array}$ & $\begin{array}{l}1 \text { verre } M \text { et } S \text { durée indéterminée } \\
1 \text { poire/j pendant } 3 \mathrm{j}\end{array}$ \\
\hline
\end{tabular}




\section{DISCUSSION}

En prenant en compte le critère genre, pour les enquêtés, les hommes ont été majoritairement représentés $(55,32 \%)$. Ce constat est dû à l'incapacité des femmes à pouvoir se rendre en forêt pour la récolte des échantillons, bien que détentrice du même savoir traditionnel. Par contre, d'autre enquêtes ethnobotaniques effectuées dans la région du littoral, notamment dans les marchés de Douala et ses environs, montrent plutôt que parmi les enquêtés, les femmes sont majoritaires (Ladoh et al., 2016). Toutes les tranches d'âge ont été concernées par les enquêtes menées et les paysans ciblés, familiers aux espèces végétales et à leur(s) utilisation(s) pour les traitements des maladies concernées. Toutefois, la tranche âgée (60-70 ans) ne semble plus intéressée par la transmission des connaissances traditionnelles. Seulement quelques jeunes ( $\leq 29$ ans), suivent les pratiques médicinales et la connaissance traditionnelle transmises par les adultes (30-59 ans). Tel est également le cas dans certaines régions du globe notamment au Népal (Joshi et Edington, 1990). La langue bassa est celle des populations visitées, et bien que quelques halogènes (parlant aussi la langue, y soient infiltrés), pour cette raison, la récolte des données a nécessité la présence d'un traducteur originaire de chaque village enquêté. L'éloignement des centres de santé des hameaux villageois, montre que l'usage traditionnel des plantes médicinales, transmis de façon héréditaire (de père en fils) et orale, constitue la base de la médecine curative de ces populations vivant dans un état de paupérisation prononcée (Cunningham, 1993). Les données collectées ont permis de recenser 94 espèces appartenant à 92 genres et à 54 familles. D'autres études ont été conduites dans le monde, en Afrique et au Cameroun sur les plantes médicinales. Les travaux portant sur la connaissance indigène des plantes ethno botanique du district de Kavrepalanchowk au Népal, a permis de recenser 68 espèces appartenant à 39 genres et 37 familles (Birendra et Chhetri, 2009). Les enquêtes ethnobotaniques portant sur la stratégie de valorisation des espèces ressources des produits non ligneux de la savane des environs de Kinshasa, ont recensés 49 espèces médicinales réparties dans 23 familles (Makumbelo et al., 2008). Des enquêtes sur les connaissances des plantes médicinales des groupes ethniques de la ville de Douala ont recensé 94 plantes appartenant à 84 genres et 46 familles (Mpondo et Dibong, 2011). L'identification botanique a montré que parmi les 54 familles recensées, celles les plus représentées sont les Fabaceae, 9 espèces, Euphorbiaceae, 6 espèces, Asteraceae, 6 espèces,
Acanthaceae, 4 espèces, et Rubiaceae, 4 espèces. D'autres études menées dans le monde, en Afrique et au Cameroun, ont permis de recenser de nombreuses familles parmi tant d'autres. Dans une région de l'Ouganda, les Asteraceae ont été recensés comme étant la famille la plus représentée (Hamill et al., 2013). $\mathrm{Au}$ Cameroun, des travaux révèlent comme familles dominantes, les Asteraceae, 6 espèces, Apocynaceae, 4 espèces et Euphorbiaceae, 4 espèces (Ngene et al., 2015). La similarité entre les familles prédominantes ailleurs et celles de la Sanaga maritime, témoignent de la richesse floristique de ce département. Les espèces de culture représentent le type de biotope majoritaire avec $28,85 \%$. Ces espèces se retrouvent domestiquées dans des agroécosystèmes, car la domestication des plantes peut s'avérer être une alternative dans les stratégies d'exploitation durable de cette ressource (Mvogo, 2013). En second plan, viennent les espèces de forêt notamment les forêts primaires $(21,15 \%)$. Ces résultats convergent avec ceux de Mabika qui recense au kasai occidental dans le parc national d'Odzala en RDC 87,7\% d'espèces de forêt primaire. Les mêmes résultats sont également obtenus dans le Dja avec une majorité d'espèces de forêt dont une prédominance à $68 \%$ d'espèces de forêt primaire. Les différences de proportions d'habitats seraient dues au climat de la région $\mathrm{du}$ Littoral qui seul, varie d'une zone à une autre ainsi que la biodiversité des sols et des forêts (Betti, 2001). Les espèces largement répandues ont représenté les types phytogéographiques dominants avec $24,04 \%$ de pantropicales et 20,19\% d'Afro-tropicales. Par contre, les travaux de Betti dans le Dja et les marchés de Yaoundé ressortent une prédominance d'espèces cosmopolites parmi les espèces largement répandues. Dans le parc national de l'lvindo au Nord-est du Gabon, la description des groupements végétaux et de la flore relève une forte proportion (30\%) des cosmopolites (Betti, 2001). Les espèces guinéo-congolaises occupent la troisième place avec $19,23 \%$. Ces résultats sont presque similaires à ceux de Betti qui trouve également dans le dja une prédominance d'espèces guinéo-congolaises avec des proportions de $41 \%$ et $68,51 \%$ d'élément de base guinéen. Dans les marchés de Yaoundé, il enregistre $48 \%$ d'espèces guinéo-congolaises et $85 \%$ d'élément de base guinéen (Betti, 2001). Au Gabon, $77 \%$ des espèces endémiques guinéo-congolaises sont recensées dans les prairies des inselbergs du nord de ce pays (Ngok, 2005). Cette dominance d'espèces guinéo congolaises traduit ainsi le faible impact des cultures étrangères sur l'exploitation des plantes médicinales et la conservation 
des cultures locales. En regroupant les plantes médicinales identifiées dans cette étude, les herbacées ont représenté le type morphologique majoritaire avec des proportions de $37,50 \%$. Ces résultats sont en accord avec ceux de Mpondo et Dibong qui ont montré que les plantes médicinales connues des différentes ethnies de Douala sont en majorité les herbes. Ce résultat se justifierait par la forte utilisation des feuilles dans les préparations et le mode de récolte manuel couramment utilisés (Mpondo et Dibong, 2012). Les arbres et les arbustes ont des proportions significatives à ne pas négliger position soit $29,81 \%$ et $27,88 \%$. Par contre, les arbres sont considérés comme les types morphologiques les plus vulnérables, ceci du fait de l'écorçage systématique des arbres à cernes pratiqué par les populations locales, provoquant ainsi leur dépérissement à plus ou moins long terme (Cunningham, 1988). L'étude menée a permis de recenser dans l'ensemble une prédominance de plantes à sarcochores $(43,27 \%)$. Ces résultats sont similaires aux travaux de Ndjib (2013) qui révèlent $57 \%$ de plantes à sarcochores. Ce type de diaspores aurait un pouvoir germinatif élevé L'importance des espèces à sarcochores montre d'une part leur origine forestière et le rôle important de la faune sauvage dans la régénération forestière $\mathrm{Ndjib}$ (2013). Les parties végétales utilisées ont été classées par ordre d'importance décroissante: les feuilles $(61,18 \%)$, les écorces $(22,35 \%)$, les fruits $(8,24 \%)$. Le reste des parties utilisées a été représenté par un taux de $7,08 \%$ à savoir $1,18 \%$ pour chacune (Bulbe, graine, sève, rhizome, tige

\section{CONCLUSION}

Les enquêtes ethnobotaniques effectuées auprès des paysans de trois arrondissements du département de la Sanaga maritime révèlent que la flore camerounaise est riche en espèces de plantes médicinales capables de soigner différentes catégories de maladies, symptômes, et traumatismes de cause externe. Quatre vingt quatorze espèces appartenant à 92 genres et 54 familles ont été dénombrés. Les principales familles sont les Fabaceae ( 9 espèces), Asteraceae (6 espèces), Euphorbiaceae (6 espèces), Acanthaceae (4 espèces) et Rubiaceae (4

\section{REFERENCES}

Awono A, Manirakiza D, Ingram V., 2009. Mobilisation et renforcement des capacités des petites et moyennes entreprises impliquées dans la filière des produits forestiers non ligneux en Afrique Centrale. CIFOR : Cameroun. http: camerounforet. com/fr/bibliotheque/10666. et tronc). La fréquence d'utilisation élevée de feuilles peut être expliquée par l'aisance et la rapidité de la récolte mais aussi par le fait qu'elles sont le siège de la photosynthèse et parfois du stockage des métabolites secondaires responsables des propriétés biologiques de la plante (Bitsindou, 1986). Quatre-vingt recettes ont été recensées et les fréquences de citations des plantes ont varié entre 1,06 et 12,77 ; ces valeurs sont relativement faibles. Preuve que les connaissances ethnobotaniques entre les villages sont disparates et s'éloignent davantage entre Ngambe (35 espèces exclusives) d'une part, Pouma (10 espèces exclusives), et Ndom (16 espèces exclusives) d'autre part. De plus, les plantes utilisées dans le traitement des maladies pour la plupart, ne sont pas courantes et pourraient contribuer à la découverte de nouveaux médicaments et combler le déficit majeur qui affecte l'industrie pharmaceutique (Bhushan, 2005). Les résultats obtenus de l'évaluation des métabolites secondaires, combinant pharmacologie et identification chimique, ont été étonnants, car confirment dans leur majorité l'indication thérapeutique recueillie, montrant la pertinence des savoirs traditionnels dans le département de la Sanaga maritime. Les plantes recensées ont été classées en deux catégories: les plantes à principes actifs isolés et les plantes à extraits standardisés, où les molécules identifiées possèderaient une légère activité toujours inférieure à celle de l'extrait. II en découle une synergie entre les différents principes, renforçant la biodisponibilité ou l'efficacité et rendant l'extrait complexe et supérieur à la somme des parties.

espèces). Les espèces de culture ont été majoritairement représentées. La phytogéographie a révélé une prédominance des espèces pantropicales avec un pourcentage de 24,04, suivies des espèces afrotropicales $(20,19 \%)$. Les espèces ligneuses (arbres, arbustes, sous arbustes et lianes) représentent $62,50 \%$, contre $37,50 \%$ d'herbacées. De ces espèces, 80 recettes de plantes ont été recensées et présentent des propriétés thérapeutiques très intéressantes.

Betti J L., 2001. Usages traditionnels et vulnérabilité des plantes médicinales dans la réserve du Dja et dans les marchés de Yaoundé, Cameroun. Thèse de Doctorat de l'Université Libre de Bruxelles. 289p. 
Betti J L., 2002. Medicinal plants sold in Yaoundé markets, Cameroon. African Study Monographs. 23 (3): 47-64.

Birendra M, Chhetri R., 2009. Indigenous knowledge on ethnobotanical plants of Kavrepalanchowk district. Journal of Science, Engineering and Technology, 5: 96-109.

Bitsindou M., 1986. Enquête sur la phytothérapie traditionnelle à Kindamba et Odzala (Congo) et analyse de convergence d'usage des plantes médicinale en Afrique centrale -Mem. Doc (inéd.). Université libre de Bruxelles. $482 \mathrm{p}$.

Bhushan P., 2005. Ethnopharmacology and drug discovery. Journal of Ethnopharmacology, 100: 50-52.

Chikamai B, Tchatat M, Tieguhong J, Ndoye O., 2009. Forest management for non-wood forest products and services in sub-saharan Africa. Discov. Innov. 1: 50-59.

Cunningham A., 1993. African medicinal plants setting priorities at the interface between conservation and primary health care. UNESCO Paris: People and Plants. Working paper n¹, pp.1-50.

Dibong S, Mpondo M, Ngoye A, Kwin N, Betti J L., 2011. Ethnobotanique et phytomédecine des plantes médicinales vendues sur les marchés de Douala, Cameroun. Journal of Applied Biosciences, 37: 2496-2407.

Fondoum J, Tiki Manga, 2000. Farmer'sindigenous practices for conserving Garcinia kola and Gnetum africanum in southern Cameroon. Agroforestry Systems, 48: 289-302.

Guedje N, Fankap R., 2001. Utilisations traditionnelles de Garcinia lucida et Garcinia kola (Clusiaceae) au Cameroun. Syst. Geogr. PI., 71: 747-758.

Hamill F, Apio S, Mubiru N, Bukenya-Ziraba R, Mosango M, Maganyi O., 2003. Traditional herbal drugs of Southern Uganda, II: literature analysis and antimicrobial assays. Journal of Ethnopharmacology, 84: 57-78.

Jiofack T, Fokunang C, Guedje N, Kemeuze V, Fongnzossie E, Nkongmeneck B., 2010. Ethnobotanical uses of medicinals plants of two ethnoecological regions of Cameroon. Int. J. of Med. and Med. Sc., 2 (3): 60-79.

Joshi A, Edington J., 1990. The use of medicinal plants by two village communities in the central development region of Nepal. Economic Botany. 44: 71-83.

Koné Kamanzi A., 2006. Inventaire ethnomédical et évaluation de l'activité antihelminthique des plantes médicinales utilisées en Côte d'Ivoire contre les helminthiases intestinales. Pharm. Méd. Trad. Afr., (14) : 55-72.

Ladoh Y, Vandi D, Dibong S D, Mpondo Mpondo E, Wansi J, Betti J L., 2016. Étude des plantes médicinales commercialisées dans les marches de la ville de Douala, Cameroun. Journal of Applied Biosciences, 99 : 9450-9466.

Letouzey R., 1970. Manuel de botanique forestière, Afrique Tropicale. Tome 2, CTFT, Sainte Marie. France.

Makumbelo E, Lukoki L, Paulus J, Luyindula, 2008. Stratégie de valorisation des espèces ressources des produits non ligneux de la savane des environs de Kinshasa: II. Enquête ethnobotanique (aspects médicaux), 26 (3): 129-134.

Mbita Messi H., 1998. Contribution à l'étude des plantes médicinales du Cameroun : Le cas des plantes utilisées en médecine traditionnelle pour le traitement des maladies parasitaires [Thèse doctorat 3è cycle]. Yaoundé : université de Yaoundé $\mathrm{l}$.

Mpondo Mpondo E, Dibong S D., 2011. Medicinal plant knowledge of ethnic group in Douala Town Cameroon. American Journal of Food and Nutrition. 1(4): 178-184.

Mpondo Mpondo E, Dibong SD., 2012. Traditional knowledge on medicinal plants use by ethnic communities in Douala, Cameroon. European Journal of Medicinal Plants. 2 (2): 159-176.

Mvogo O., 2013. Ethnobotanique et phytomédecine des plantes à flavonoides vendues dans les marchés de la ville de Douala. Mémoire de Diplôme d'Etudes Approfondies de l'Université de Douala. 54p.

Ndjib R C., 2013. Ethnobotanique des plantes à huiles essentielles commercialisées dans deux marchés de Douala. Mémoire de Diplôme d'Études Approfondies de l'Université de Douala. $85 \mathrm{p}$.

Ngene J, Ngoule C, Kidik P, Mvogo O, Ndjib R, Dibong S D, Mpondo Mpondo E. 2015. Importance de la pharmacopée traditionnelle des plantes à flavanoides vendues dans les marchés de Douala est (Cameroun). Journal of Applied Biosciences, 88: 8194-8210.

Ngok B., 2005. Diversité végétale des inselbergs et des dalles rocheuses du Nord du Gabon. Thèse de doctorat, Université libre de Bruxelles. 420p. 
Priso R, Nnanga J, Etame J, Din N, Amougou A., 2011.

Les produits forestiers non ligneux d'origine végétale: valeur et importance dans quelques marchés de la région du Littoral-Cameroun. Journal of Applied Biosciences. $40: 2715-2726$.

Schnell R., 1960. Techniques d'herborisation et de conservation des plantes dans les pays tropicaux. Journal of Agricutural and Tropical Botanical Applied, 7 (1-3): 1-48. 